\title{
THE NUMBER OF RATIONAL QUARTICS ON CALABI-YAU HYPERSURFACES IN WEIGHTED PROJECTIVE SPACE P $\left(2,1^{4}\right)$
}

\author{
PAUL MEURER
}

\section{Abstract.}

We compute the number of rational quartics on a general Calabi-Yau hypersurface in weighted projective space $P\left(2,1^{4}\right)$. The result agrees with the prediction made by mirror symmetry.

\section{Introduction.}

In this note we will compute the number of rational quartics (see §3) on a general Calabi-Yau hypersurface in weighted projective space $P\left(2,1^{4}\right)$. The number was asked for for the first time by Sheldon Katz [8], and David Morrison [10] computed it using mirror symmetry methods. Our result, which agrees with the mirror symmetry computation, is:

THeOREM 1.1. There are 6028452 rational quartics on a general Calabi-Yau hypersurface in weighted projective space $\mathrm{P}\left(2,1^{4}\right)$.

The method used can be sketched as follows: We show that the irreducible component $\tilde{\mathscr{H}}_{4}$ of the Hilbert scheme of $\mathrm{P}\left(2,1^{4}\right)$ containing the rational quartics is smooth and can be embedded into the irreducible component $\mathscr{H}_{4}$ of the Hilbert scheme of $\mathrm{P}^{4}$ containing the elliptic quartic curves. There, it can be characterized as one component of the fixed point scheme of a natural involution. On the other hand, $\mathscr{H}_{4}$ is well-known and explicitly described by Dan Avritzer and Israel Vainsencher [1]. Together, this leads to an explicit description of $\tilde{\mathscr{H}}_{4}$.

The number of rational quartics on a general Calabi-Yau hypersurface is given as the integral of the top Chern class of a certain vector bundle on $\tilde{\mathscr{H}}_{4}$. It will be computed by a formula of Bott's, which expresses the integral of a homogeneous polynomial in the Chern classes of a bundle on a smooth, compact variety with a $C^{*}$-action in terms of data given by the induced linear actions on the fibers of the bundle and the tangent bundle in the (isolated) fixed points of the action.

Received September 7, 1994. 
ACKNOWLedgement. I would like to express my thanks to Stein Arild Strømme for many helpful conversations.

\section{Relations between the Hilbert schemes of $X$ and $X / \Gamma$.}

In this section, we investigate relations between the Hilbert schemes of a projective scheme $X$ and its quotient $X / \Gamma=Y$ w.r.t. the action of a finite group $\Gamma$. To each irreducible component $\mathscr{H}$ of $\mathbf{H i l b}_{X / \Gamma}$, we will find a subscheme $Z$ of $\mathbf{H i l b}_{X}$ mapping birationally onto $\mathscr{H}$. The morphism $\varphi: Z \rightarrow \mathscr{H}$ is generally no isomorphism, but in the situation interesting us primarily (i.e. $\mathscr{H}=$ irreducible component of Hilb $_{\mathrm{P}\left(2,1^{4}\right)}$ containing the rational quartics), it actually is an isomorphism.

We assume all schemes to be defined over an algebraically closed field $k$ of characteristic 0 .

First, we state some nice simple properties of finite quotients $\rho: X \rightarrow X / \Gamma=Y$ for later reference.

LEMMA 2.1. (i) $\rho_{*}$ and $\left(\rho_{*}(\cdot)\right)^{\Gamma}$ are exact functors from $\Gamma$-linearized quasicoherent $\mathcal{O}_{X}$-modules to quasicoherent $\mathcal{O}_{Y}$-modules.

(ii) Let $V \subseteq X$ be $\Gamma$-invariant. Then we have

$$
\mathcal{O}_{V / \Gamma}=\left(\rho_{*} \mathcal{O}_{V}\right)^{\Gamma}, \quad \mathscr{I}_{V / \Gamma}=\left(\rho_{*} \mathscr{I}_{V}\right)^{\Gamma} .
$$

(iii) $\left(\rho_{*}(\cdot)\right)^{r}$ commutes with cohomology, i.e.

$$
H^{i}(X, \mathscr{F})^{\Gamma}=H^{i}\left(Y,\left(\rho_{*} \mathscr{F}\right)^{\Gamma}\right)
$$

ProOF. (i) $\rho$ is a finite map.

(ii) follows immediately from the definition of $V / \Gamma$ and (i).

(iii) Note that the functors $H^{i}(X, \cdot)^{\Gamma}$ and $H^{i}\left(Y,\left(\rho_{*}(\cdot)\right)^{\Gamma}\right)$ are left-exact and equal for $i=0$. But the category of quasicoherent $\mathcal{O}_{X}$-modules has enough injectives, so they are equal for all $i$.

LEMMA 2.2. Let $X$ be a quasiprojective scheme and $\Gamma$ a finite group acting on $X$. Let

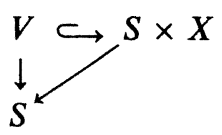

be a family of subschemes of $X$, flat over $S$ and invariant under the action of $\Gamma$. Then $V / \Gamma \subseteq S \times X / \Gamma$ is flat over $S$.

Proof. We can assume that $S$ and $X$ are affine, since flatness is a local property and $X \rightarrow Y$ is an affine map. Write $S=\operatorname{Spec} A, X=\operatorname{Spec} R_{0}$, $R=A \otimes R_{0}$. On every $R$-module $M$, we define an $R^{\Gamma}$-linear endomorphism $\Phi_{M}: M \rightarrow M$ by 


$$
\Phi_{M}(x):=|\Gamma|^{-1} \sum_{\gamma \in \Gamma} \gamma(x) .
$$

Then $M^{\Gamma}=\Phi_{M}(M)$ splits as $M=M^{\Gamma} \oplus \operatorname{ker} \Phi_{M}$. In particular, we have $\mathcal{O}_{V}=\tilde{M}$ for some $A$-flat $R$-module $M$, so $\mathscr{O}_{V / \Gamma}=\tilde{M}^{\Gamma}$, and $M^{\Gamma}$ is $A$-flat as a direct summand of a flat $A$-module.

For a finite group $\Gamma$ acting on a quasiprojective scheme $H$, we can define the fixed point scheme $H^{\Gamma}$ of the $\Gamma$-action as follows:

If $H=\operatorname{Spec} R$ is affine, then $H^{\Gamma}$ is defined by the ideal generated by $\operatorname{ker}\left(\Phi_{R}\right)$. In general, cover $H$ by affine invariant open sets $U$ and let $\left(H^{r}\right)_{\mid U}:=U^{\Gamma}$.

$H^{\Gamma}$ can be characterized as the maximal subscheme of $H$ all of whose sections are invariant under $\Gamma$.

Note that the group $\Gamma$ acts in a natural way on $\mathbf{H i l b}_{X}$ : if $[C] \in \mathbf{H i l b}_{X}$ and $\gamma \in \Gamma$, the action is given by $\gamma([C]):=[\gamma(C)]$.

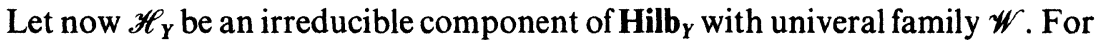
any open set $U \subseteq \mathscr{H}_{Y}$, denote by $\mathscr{W}_{U}$ the restriction of $\mathscr{W}$ to $U$ and let $\rho_{U}:=\rho \times \mathrm{id}_{U}$. The lift of the family $\mathscr{W}_{U}$ to $X$ is given by $\rho_{U}^{-1} \mathscr{W}_{U}=$ $\mathscr{W}_{U} \times{ }_{Y \times U} X \times U$.

THEOREM 2.3. Suppose that there exists an open subset $U \subseteq \mathscr{H}_{Y}$ such that $\rho_{U}^{-1} \mathscr{W}_{U}$ is flat. Then there is a uniquely determined irreducible component $Z$ of $\left(\mathbf{H i l b}_{X}\right)^{T}$ mapping birationally to $\mathscr{H}_{Y}$ by the map $[C] \mapsto[C / \Gamma]$.

Remark. The existence of such an open set is guaranteed for example if $\mathscr{H}_{Y}$ is reduced (cf. [11], Lect. 8: Flattening stratifications).

PROOF. $\rho_{\mathscr{H}_{Y}}^{-1} \mathscr{W}$ is in general not flat over $\mathscr{H}_{Y}$, but by assumption, there is an open subset $U \subseteq \mathscr{H}_{Y}$ such that $\rho_{U}^{-1} \mathscr{W}_{U}$ is flat. We can assume that $U=\operatorname{Spec} A$. By the universal property of the Hilbert functor, we thus get a morphism $\psi: U \rightarrow \mathscr{H}_{X}$, where $\mathscr{H}_{X}$ is some irreducible component of Hilb . Moreover, $\psi$ factors through an irreducible component $Z$ of $\left(\mathscr{H}_{X}\right)^{r}$. Let $\mathscr{V}_{Z} \subseteq X \times Z$ be the restriction of the universal family on $\mathscr{H}_{X}$ to $Z$.

If we denote $\mathrm{id}_{X} \times \psi$ by $\psi_{X}$ etc., it is immediate that $\rho_{U_{*}} \psi_{X}^{*} \mathscr{F}=\psi_{Y}^{*} \rho_{Z_{*}} \mathscr{F}$ for any coherent sheaf $\mathscr{F}$ on $X \times Z$. On the other hand, $\mathcal{O}_{W_{U}}=\left(\rho_{U *} \rho_{U}^{*} \mathcal{O}_{W_{U}}\right)^{\Gamma}$; a simple computation shows then that $\mathscr{W}_{U}$ is the pullback of $\mathscr{V}_{Z} / \Gamma$ by $\psi$.

By Lemma $2.2, \mathscr{V}_{Z} / \Gamma \subseteq Y \times Z$ is flat over $Z$, therefore we get a morphism $\varphi$ from $Z$ to the same component $\mathscr{H}_{Y}$ such that $\mathscr{V}_{Z} / \Gamma$ is the pullback of the universal family $\mathscr{W}_{\mathbf{Y}}$.

Since $\rho_{U}^{-1} \mathscr{W}_{U}$ is flat over $U$, it is again easy to see that $\mathscr{V}_{\varphi^{-1} U}$ is the pullback of $\rho_{U}^{-1} \mathscr{W}_{U}$ by $\varphi$.

We can summarize the situation in the following commutative diagram: 


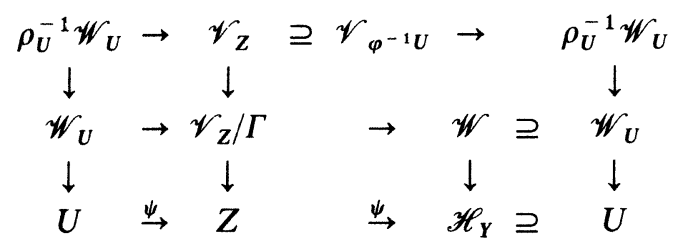

Since all maps in the first and second rows are pullbacks by $\psi$ or $\varphi$, it follows by the universal property of the Hilbert functor that $\varphi \circ \psi=\mathrm{id}_{U}$ and $\psi \circ \varphi_{\varphi^{-1} U}=$ id $_{\varphi^{-1} U}$. Therefore, $\varphi: Z \rightarrow \mathscr{H}_{Y}$ is birational.

EXAMPLE. A simple example showing that the map $\varphi: Z \rightarrow \mathscr{H}_{Y}$ doesn't need to be an isomorphism is the following:

Take $X=\mathrm{P}^{2}$ and $\Gamma=\{$ id, $\imath\}$, where $\Gamma$ acts on $\mathrm{P}^{2}$ by $\imath x_{0}=-x_{0}$, and $\imath x_{i}=x_{i}$ for $i=1,2$. Then $Y=X / \Gamma$ is the weighted projective space $\mathrm{P}(2,1,1)$ (cf. Definition 3.1). Let $\mathscr{H}_{\mathrm{P}(2,1,1)}^{1}$ be the Hilbert scheme of subschemes of length 1 in $P(2,1,1)$, which is isomorphic to $P(2,1,1)$ itself, and singular in the point $(1,0,0)$. On the other hand, since the inverse image of a general point in $\mathrm{P}(2,1,1)$ is a pair of points in $\mathrm{P}^{2}, Z$ lies in the Hilbert scheme $\mathscr{H}_{\mathrm{P}^{2}}^{2}$ of subschemes of length 2 in $\mathrm{P}^{2}$, which is a $\mathrm{P}^{2}$-bundle over $\breve{\mathrm{P}}^{2}$, hence smooth. By Corollary $2.5, Z$ is smooth too, hence $Z$ can't be isomorphic to $P(2,1,1)$. In fact, $Z$ is the blow up of $P(2,1,1)$ in the singular point.

The following proposition is certainly well known. Since we didn't find a reference for it, we give a proof.

Proposition 2.4. Let $H$ be a smooth scheme and $\Gamma$ a finite group acting on $H$.

(i) The fixed point scheme $H^{\Gamma}$ of $\Gamma$ is a smooth subscheme of $H$.

(ii) The Zariski tangent space $\mathscr{T}_{H^{r}}(x)$ to $H^{\Gamma}$ in a point $x$ is equal to $\mathscr{T}_{H}(x)^{\Gamma}$.

Proof. (i) Let $p \in H^{\Gamma}$ be a closed point and $R=\mathcal{O}_{p, X}$; consider the induced action of $\Gamma$ on $R$. Then the ideal $\mathrm{a}_{\Gamma}$ of $H^{\Gamma}$ in $R$ is generated by ker $\Phi_{R}$.

Since $H$ is smooth, $R$ is a regular (noetherian) local ring. We will show that also $R / a_{\Gamma}$ is regular, which proves the proposition.

Let $\mathbf{m} \subseteq R$ be the maximal ideal. $\Gamma$ induces a linear action on the vector space $\mathbf{m} / \mathbf{m}^{2}=V$.

Let $\hat{x}_{1}, \ldots, \hat{x}_{d}$ be a base of the subspace of $V$ invariant under $\Gamma$, and complete it by $\hat{y}_{1}, \ldots, \hat{y}_{n-d}$ to a base of $V$, such that the $\hat{y}_{j}$ satisfy $\Phi_{R}\left(\hat{y}_{j}\right)=0$. This can be achieved by choosing arbitrary $y_{j}$ and setting $\hat{y}_{j}=y_{j}-\Phi_{R}\left(y_{j}\right)$. By Nakayama's lemma, the $\hat{x}_{i}, \hat{y}_{j}$ lift to generators $\tilde{x}_{i}, \tilde{y}_{j}$ of $\mathbf{m}$.

Again we can construct new ring elements by averaging: Let $x_{i}:=\Phi_{R}\left(\tilde{x}_{i}\right)$, $y_{j}:=\tilde{y}_{j}-\Phi_{R}\left(\tilde{y}_{j}\right)$. The images of $x_{i}, y_{j}$ in $R / \mathrm{m}^{2}$ are $\hat{x}_{i}, \hat{y}_{j}$. We conclude that also $x_{i}$, $y_{j}$ generate $\mathbf{m}$. We will show that $\mathbf{a}:=\left(y_{1}, \ldots, y_{n-d}\right)$ is equal to $\mathbf{a}_{\Gamma}$; then $R / \mathbf{a}_{\Gamma}$ is a regular local ring, and $H^{r}$ is smooth. 
The ideal $\mathbf{a}$ is clearly contained in $\mathbf{a}_{\Gamma}=\left(\operatorname{ker} \Phi_{R}\right)$. On the other hand, suppose that

$$
\operatorname{ker} \Phi_{R} \subseteq \mathbf{a}+\mathbf{m}^{r},
$$

(this is trivially true for $r=1$ ), and let $b=\sum_{i} \alpha_{i} x_{i}+\sum_{j} \beta_{j} y_{j} \in \operatorname{ker} \Phi_{R}$ be an arbitrary element. We can write

$$
b=b-\Phi_{R}(b)=\sum\left(\alpha_{i}-\Phi_{R}\left(\alpha_{i}\right)\right) x_{i}+\sum \beta_{j} y_{j}-\Phi_{R}\left(\beta_{j} y_{j}\right) .
$$

The first sum is contained in $\mathbf{a}+\mathbf{m}^{r+1}$ because $\alpha_{i}-\Phi_{R}\left(\alpha_{i}\right)$ lies in $\operatorname{ker} \Phi_{R} \subseteq$ $\mathbf{a}+\mathbf{m}^{r}$. The summands $\beta_{j} y_{j}$ lie in a. Write now

$$
\begin{aligned}
\Phi_{R}\left(\beta_{j} y_{j}\right) & =\Phi_{R}\left(\Phi_{R}\left(\beta_{j}\right) y_{j}+\left(\beta_{j}-\Phi_{R}\left(\beta_{j}\right)\right) y_{j}\right) \\
& =\Phi_{R}\left(\left(\beta_{j}-\Phi_{R}\left(\beta_{j}\right)\right) y_{j}\right) \\
& =|\Gamma|^{-1} \sum_{\gamma} \gamma\left(\beta_{j}-\Phi_{R}\left(\beta_{j}\right)\right) \gamma\left(y_{j}\right) .
\end{aligned}
$$

In the last sum, both factors of the summands lie in $\operatorname{ker} \Phi_{R}$, hence $\Phi_{R}\left(\beta_{j} y_{j}\right)$ lies in $\mathbf{a}+\mathbf{m}^{r+1}$, too, and together we have that $b \in \mathbf{a}+\mathbf{m}^{r+1}$, hence

$$
\operatorname{ker} \Phi_{R} \subseteq \mathbf{a}+\mathbf{m}^{r+1} \text {. }
$$

Since $\bigcap_{r} \mathbf{m}^{r}=\emptyset$, we get by induction on $r$ that

$$
\operatorname{ker} \Phi_{R} \subseteq \mathbf{a} .
$$

Hence $\mathbf{a}_{\Gamma}=\alpha$, and $H^{\Gamma}$ is smooth.

(ii) is immediate.

With the notation of Theorem 2.3, we have

Corollary 2.5. $Z$ is smooth if $\mathscr{H}_{X}$ is smooth.

\section{Rational quartics in $P\left(2,1^{4}\right)$ and elliptic quartics in $P^{4}$.}

DEFINITION 3.1. A weighted projective $n$-space $\mathrm{P}\left(k_{0}, \ldots, k_{n}\right)$ with positive integer weights $k_{0}, \ldots, k_{n}$ is defined as Proj of the graded ring $R:=k\left[y_{0}, \ldots, y_{n}\right]$, the variables $y_{0}, \ldots, y_{n}$ having weights $k_{0}, \ldots, k_{n}$. We can assume that the $k_{0}, \ldots, k_{n}$ are coprimal.

There is an isomorphism of graded rings $R \cong k\left[x_{0}^{k_{0}}, \ldots, x_{n}^{k_{n}}\right]$, the $x_{i}$ having weight 1 , and in the following we will work with the $x_{i}$ rather than the $y_{i}$.

We will use the abbreviation $\tilde{\mathbf{P}}$ for an arbitrary weighted projective space.

Let $\Gamma_{k}$ be the group of $k$ th roots of unity, and let $\Gamma:=\Gamma_{k_{0}} \times \ldots \times \Gamma_{k_{n}}$. $\Gamma$ acts on $\mathrm{P}^{n}=$ Proj $k\left[x_{0}, \ldots, x_{n}\right]$ in the obvious way, namely through the action of $\Gamma_{\boldsymbol{k}_{\boldsymbol{t}}}$ on the $i$-th projective coordinate by multiplication. $\tilde{P}$ can then be described as the geometric quotient of $\mathrm{P}^{n}$ by this action of $\Gamma$ : 


$$
\mathrm{P}\left(k_{0}, \ldots, k_{n}\right)=\mathrm{P}^{n} / \Gamma \text {. }
$$

We denote by $\rho$ the quotient map $\mathrm{P}^{n} \rightarrow \tilde{\mathrm{P}}$, which is a finite ramified covering map, induced by the inclusion $k\left[x_{0}^{k_{0}}, \ldots, x_{n}^{k_{n}}\right] \subset k\left[x_{0}, \ldots, x_{n}\right]$. The ramification locus of $\rho$ is the union of the fixed point sets of the non-identity elements of $\Gamma$.

The singular points of $\tilde{P}$ are exactly the points $(0, \ldots, 0,1,0, \ldots, 0)$ such that $k_{i}>1$.

We can define twisting sheaves in the usual way by setting $\mathcal{O}_{\tilde{p}}(r):=R(r) \sim$. In general, these sheaves need not to be invertible, but when $\widetilde{\mathrm{P}}=\mathrm{P}\left(k, 1^{n}\right)$, then $\mathcal{O}_{\tilde{\mathrm{P}}}(k)$ is a very ample line bundle and induces a Veronese type embedding of $\tilde{P}$ into a big projective space $\mathrm{P}^{N}$ : the image of $\widetilde{\mathrm{P}}$ is the projective cone over the image $v_{k}\left(\mathrm{P}^{n-1}\right)$ of the Veronese embedding of $\mathrm{P}^{n-1}$ by $\mathcal{O}_{p^{n-1}}(k)$.

From now on we will consider only the case $\mathbb{P}=\mathrm{P}\left(k, 1^{n}\right)$.

There is a natural projection from the singular point onto $P^{n-1}$ induced by the inclusion $k\left[x_{1}, \ldots, x_{n}\right] \subset k\left[x_{0}^{k}, x_{1}, \ldots, x_{n}\right]$. In $\mathrm{P}^{N}$, this projection is given by projecting the cone down to $v_{k}\left(\mathrm{P}^{n-1}\right)$ from the point $(1,0, \ldots, 0)$.

We will need the following

Lemma 3.1. The quotient map $\rho: \mathrm{P}^{n} \rightarrow \mathrm{P}\left(k, 1^{n}\right)$ is flat away from the singular point $(1,0, \ldots, 0)$.

Proof. $\mathrm{P}^{n}$ can be covered by the $\Gamma$-invariant affine open sets $D_{+}\left(x_{i}\right)$, $i=0, \ldots, n$, and the union $\bigcup_{i=1}^{n} D_{+}\left(x_{i}\right)$ is equal to $\mathrm{P}^{n}-\{(1,0, \ldots, 0)\}$. It suffices to show that all the maps $D_{+}\left(x_{i}\right) \rightarrow D_{+}\left(x_{i}\right) / \Gamma$ are flat for $i=1, \ldots, n$. Let

$$
R_{i}=k\left[\frac{x_{0}}{x_{i}}, \ldots, \frac{x_{i-1}}{x_{i}}, \frac{x_{i+1}}{x_{i}}, \ldots, \frac{x_{n}}{x_{i}}\right] .
$$

Then the invariant ring is

$$
R_{i}^{\Gamma}=k\left[\left(\frac{x_{0}}{x_{i}}\right)^{k}, \frac{x_{1}}{x_{i}}, \ldots, \frac{x_{i-1}}{x_{i}}, \frac{x_{i+1}}{x_{i}}, \ldots, \frac{x_{n}}{x_{i}}\right],
$$

and we have $D_{+}\left(x_{i}\right)=\operatorname{Spec} R_{i}, D_{+}\left(x_{i}\right) / \Gamma=\operatorname{Spec} R_{i}^{\Gamma}$. Thus the ring $R_{i}$ is equal to $\oplus_{s=0}^{k-1}\left(\frac{x_{0}}{x_{i}}\right)^{s} \cdot R_{i}^{\Gamma}$, hence a free $R_{i}^{\Gamma}$-module, and $D_{+}\left(x_{i}\right)$ is flat over $D_{+}\left(x_{i}\right) / \Gamma$.

Corollary 3.2. Let $V \subset S \times \mathrm{P}\left(k, 1^{n}\right)$ be a flat family over $S$ such that no fiber of $V$ contains the singular point of $V$. Then $\rho_{S}^{-1} V \subseteq S \times \mathrm{P}^{n}$ is flat over $S$.

Proof. $V$ is a flat family in $\mathrm{P}\left(k, 1^{n}\right)-\{(1,0, \ldots, 0)\}$. The claim follows from the lemma and by transitivity and base change stability of flatness.

There is no intrinsic notion of degree of a curve in a weighted projective space P. In the case of a weighted projective space of type $\widetilde{P}=P\left(k, 1^{n}\right)$, however, we 
agree to measure the degree of curves (or, more generally, the Hilbert polynomial of subschemes) w.r.t. the embedding described above, i.e. $\operatorname{deg} C=\operatorname{deg}\left(\mathcal{O}_{\mathrm{P}}(k)_{\mid \mathrm{C}}\right)$.

Let us now specialize to the case $\widetilde{P}=P\left(2,1^{4}\right)$, the case we are primarily interested in. $\tilde{P}=\operatorname{Proj}\left(k\left[x_{0}^{2}, x_{1}, \ldots, x_{4}\right]\right)$ is the quotient of $\mathrm{P}^{4}$ by the group $\Gamma=\{1, l\} \cong \mathrm{Z}_{2}$, the action of $\Gamma$ being given by $l x_{0}=-x_{0}, l x_{i}=x_{i}$ for $i=1, \ldots, 4$.

We will consider rational quartics, i.e. rational curves of degree (in the above sense) four in $\widetilde{\mathrm{P}}$. Let $C \subseteq \widetilde{\mathrm{P}}$ be a rational quartic and

$$
\begin{aligned}
\mathrm{P}^{1} & \rightarrow C \\
(s, t) & \mapsto\left(f_{0}(s, t), \ldots, f_{4}(s, t)\right)
\end{aligned}
$$

a parametrization of $C$. The image of $C$ under the embedding $\tilde{\mathrm{P}} \hookrightarrow \mathrm{P}^{N}$ induced from $\mathcal{O}_{\tilde{P}}(2)$ is parametrized as

$$
(s, t) \mapsto\left(f_{0}, f_{1}^{2}, f_{1} f_{2}, \ldots, f_{4}^{2}\right)
$$

and is a degree four curve by definition. Hence $f_{0}$ has degree four and $f_{1}, \ldots, f_{4}$ have degree two. Furthermore, we see that the projection of a rational quartic from the singular point to $\mathrm{P}^{3}$ is a conic in $\mathrm{P}^{3}$.

We denote by $\tilde{\mathscr{H}}_{4}$ the irreducible component of Hilb $\tilde{p}_{\tilde{p}}$ containing the rational quartics. (To see that the rational quartics are really contained in one irreducible component, observe that the parameter space of rational quartic curves (without degenerations) is fibered over the space of conics in $P^{3}$ (which is irreducible). Each fiber is irreducible, too, as one can see from (3.1) by specifying a parametrization $\left(f_{1}, \ldots, f_{4}\right)$ of a conic and letting $f_{0}$ vary. Hence the space of rational quartics is irreducible, and $\tilde{\mathscr{H}}_{4}$ is the irreducible component of Hilb pr $_{\tilde{P}}$ containing that space.) The universal curve on $\tilde{\mathscr{H}}_{4}$ will be denoted by $\mathscr{C}$.

Let now $C$ be a general rational quartic in $\tilde{\mathscr{H}}_{4}$. The quotient map $\rho^{-1} C \rightarrow$ $\rho^{-1} C / \Gamma=C$ exhibits $\rho^{-1} C$ as a double cover of $C$, and the ramification locus consists of the four points where $f_{0}=0$ in the parametrization (3.1). By Hurwitz's theorem $\rho^{-1} C$ is an elliptic curve, and it is again a quartic because $\rho^{-1} C$ intersects the hyperplane $\left\{x_{0}=0\right\}$ in four points.

Since $C$ doesn't contain the singular point of $\widetilde{P}$, there is an open subset $U \subseteq \tilde{\mathscr{H}}_{4}$ containing $[C]$ such that no fiber of $\mathscr{C}_{U}$ contains the singular point. By Corollary $3.2, \mathscr{C}_{U}$ lifts to a flat family $\rho_{U}^{-1} \mathscr{C}_{U}$ in $\mathrm{P}^{4}$.

Denote by $\mathscr{H}_{4}$ the component of Hilb $\mathrm{p}^{4}$ parametrizing the smooth elliptic quartic curves in $\mathrm{P}^{4}$ and their degenerations. According to Theorem 2.3, there is an irreducible subscheme $Z_{4}$ of $\mathscr{H}_{4}$ mapping birationally to $\tilde{\mathscr{H}}_{4}$ by $\left[C^{\prime}\right] \mapsto$ $\left[\rho\left(C^{\prime}\right)\right]$. Since $\mathscr{H}_{4}$ is smooth (see below), it follows by Corollary 2.5 that $Z_{4}$ is smooth, too.

REMARK. It is clear that the above considerations are valid as well for rational quartics in $\mathrm{P}\left(2,1^{3}\right)$ instead of $\mathrm{P}\left(2,1^{4}\right)$. Thus, if we denote by $\mathscr{H}_{3}$ the irreducible 
component of Hilb $_{p^{3}}$ parametrizing the elliptic quartic curves in $\mathrm{P}^{3}$, there is a smooth irreducible subscheme $Z_{3}$ of $\mathscr{H}_{3}$ mapping birationally to $\tilde{\mathscr{H}}_{3}$ by $\left[C^{\prime}\right] \mapsto\left[\rho\left(C^{\prime}\right)\right]$.

In order to obtain the explicit description of $Z_{4}$ that we need for the calculations, and particularly to prove the claimed isomorphy of $Z_{4}$ and $\tilde{\mathscr{H}}_{4}$, we will have to look at the description of $\mathscr{H}_{4}$ given by Dan Avritzer and Israel Vainsencher:

Let $G:=\operatorname{Grass}_{2}\left(H^{0}\left(\mathcal{O}_{\mathrm{p} 3}(2)\right)\right)$ be the Grassmannian of pencils of quadric surfaces in $\mathrm{P}^{3}$, and denote by $G^{\prime}$ the image of the (well-defined) map

$$
\begin{aligned}
\mathscr{H}_{3} & \rightarrow \operatorname{Grass}_{8}\left(H^{0}\left(\mathcal{O}_{\mathrm{P}^{3}}(3)\right)\right. \\
{[C] } & \mapsto\left[H^{0}\left(\mathscr{I}_{C}(3)\right)\right] .
\end{aligned}
$$

On $G$, we have a canonical family of subschemes of $\mathrm{P}^{3}$ : the fiber in a point $g \in G$ is the base locus of the pencil represented by $g$. In the same way, $G^{\prime}$ gives rise to a family of subschemes of $\mathrm{P}^{3}$ : the fiber in a point $g^{\prime}$ is the base locus of the linear system of cubic surfaces represented by $g^{\prime}$.

Denote by $B$ the subscheme of $G$ where the family on $G$ is not flat, and denote by $D$ the subscheme of $G^{\prime}$ where the family defined by $G^{\prime}$ is not flat ( $B$ consists of pencils with a fixed component, and $D$ is the scheme of planes in $\mathrm{P}^{3}$ with an embedded subscheme of length 2). Then we have:

THEOREM 3.3. (i) (Avritzer, Vainsencher [1]). $\mathscr{H}_{3}$ is isomorphic to a two-fold blow up of $G$. More precisely, $G^{\prime}$ is isomorphic to the blow up of $G$ along $B$ and $\mathscr{H}_{3}$ is isomorphic to the blow up of $G^{\prime}$ along $D$. The ideal of every (degenerated) curve in $\mathscr{H}_{3}$ is generated in degree $\leqq 4$. In particular, $\mathscr{H}_{3}$ is smooth of dimension 16.

(ii) $\mathscr{H}_{4}$ is fibered locally trivially over $\breve{\mathrm{P}}^{4}$ in a natural way with fiber $\mathscr{H}_{3}$; i.e., the restriction of the universal curve over $\mathscr{H}_{4}$ to the fiber over a hyperplane $h \cong \mathrm{P}^{3}$ in $\mathrm{P}^{4}$ is the universal elliptic quartic curve in $h \cong \mathrm{P}^{3}$.

Proof. (ii) We have to show that all degenerations of elliptic quartic curves in $\mathrm{P}^{4}$ span exactly a $\mathrm{P}^{3}$. Then the fibration is given by projecting a point $[C] \in \mathscr{H}_{4}$ to the hyperplane it spans.

It is clear that no degeneration can possibly span less than a $\mathrm{P}^{3}$, for then this degeneration would already be contained in $\mathscr{H}_{3}$. On the other hand, for a general elliptic curve, $\operatorname{dim}\left(H^{0}\left(\mathscr{I}_{C}(1)\right)\right)=1$; if a degeneration $C_{0}$ spanned all of $\mathrm{P}^{4}$, then $\operatorname{dim}\left(H^{0}\left(\mathscr{I}_{C}(1)\right)\right)$ would drop to 0 in $C=C_{0}$, in contradiction to upper semicontinuity of $\operatorname{dim}\left(H^{0}\left(\mathscr{I}_{C}(1)\right)\right)$.

The fibration is locally trivial because PGL(4) acts transitively on $\mathrm{P}^{4}$ and this action lifts to an action on $\mathscr{H}_{4}$. 
As a corollary of this theorem we are now able to derive an analogous description of $Z_{3}$ and $Z_{4}$. Consider therefore the inclusion of grassmannians

$$
\tilde{G}:=\operatorname{Grass}_{2}\left(H^{0}\left(\mathcal{O}_{\mathrm{P}\left(2,1^{3}\right)}(2)\right)\right) \subset G=\operatorname{Grass}_{2}\left(H^{0}\left(\mathcal{O}_{\mathrm{P}^{3}}(2)\right)\right)
$$

induced by the natural inclusion

$$
i: H^{0}\left(\mathcal{O}_{\mathrm{P}\left(2,1^{3}\right)}(2)\right) \subset H^{0}\left(\mathcal{O}_{\mathrm{P} 3}(2)\right) \text {. }
$$

Proposition 3.4. $Z_{3}$ is smooth of dimension 10 and isomorphic to the proper transform of $\tilde{G}$ under the twofold blow up map b: $\mathscr{H}_{3} \rightarrow G$.

Proof. $\Gamma$ acts on $G$. If $Z^{\prime}$ is an irreducible component of $G^{T}$ not contained in the blow up locus $B$, then the proper transform of $Z^{\prime}$ is an irreducible component of $\mathscr{H}_{3}^{\Gamma}$. On the other hand, it is easy to see that $\tilde{G} \subseteq G$ is a component of $G^{\Gamma}$. Furthermore, curves in $\tilde{G}-B$ map to rational quartics; this proves that the proper transform of $\tilde{G}$ in $\mathscr{H}_{3}$ is the right component, i.e. equal to $Z_{3}$.

We turn to the explicit description of $Z_{3}$ as a twofold blow up of $\tilde{G}$.

A pencil in $\tilde{B}=\tilde{G} \cap B$ is a pencil generated by two quadratic polynomials $F_{1}$, $F_{2}$ in $H^{0}\left(\mathcal{O}_{\mathrm{P}\left(2,1^{3}\right)}(2)\right)$ with a common linear factor, thus we have $F_{1}=f_{1} g$, $F_{2}=f_{2} g$, and they must be independent of $x_{0}$. It follows that the scheme described by such a pencil projects to the union of a line and a point in $\mathrm{P}^{2} \cong\left\{x_{0}=0\right\}$ (or a degeneration thereof) under projection from the singular point. The image is described by the same equations $F_{1}=f_{1} g, F_{2}=f_{2} g$. We conclude that $\widetilde{B}$ is isomorphic to $\breve{P} \times \mathrm{P}^{2}$ and has dimension 4 .

According to [1], a plane in $\mathrm{P}^{3}$ with an embedded subscheme of length 2 has ideal

$$
\left(x_{1}\left(x_{1}-x_{4}\right) x_{3}, x_{2} x_{3}, x_{3}^{2}\right) \text { or }\left(x_{1}^{2} x_{3}, x_{2} x_{3}, x_{3}^{2}\right)
$$

up to projective equivalence, depending on whether the subscheme has support in two points or one point. Consequently, ideals in $\tilde{D}$, the intersection of $D$ with the proper transform $\widetilde{G}^{\prime}$ of $\tilde{G}$, have, up to $\Gamma$-invariant projective equivalence, the form

$$
\left(x_{3}\left(a x_{0}^{2}+b x_{1}^{2}\right), x_{3} x_{2}, x_{3}^{2}\right), \quad(a, b) \in \mathrm{P}^{1} .
$$

Geometrically, $\tilde{D}$ parametrizes hyperplanes through $(1,0,0,0)$ with an embedded $\Gamma$-invariant subscheme of length 2 . By projecting the hyperplane down to $\mathrm{P}^{2} \cong\left\{x_{0}=0\right\}$, we see that $\tilde{D}$ is fibered over $\tilde{P}^{2}$. If we denote by $F$ the fiber over the point $\left\{x_{3}=0\right\}$, then $F$ can be covered by two smooth open sets isomorphic to $P^{1} \times A^{1}$ :

$$
\begin{aligned}
& \mathrm{P}^{1} \times \mathrm{A}^{1} \rightarrow F \\
& (a, b) \times s \mapsto\left(x_{3}\left(a x_{0}^{2}-b\left(x_{1}+s x_{2}\right)^{2}\right), x_{3}\left(s x_{1}-x_{2}\right), x_{3}^{2}\right)
\end{aligned}
$$


and

$$
\begin{aligned}
& \mathrm{P}^{1} \times \mathrm{A}^{1} \rightarrow F \\
& \left(a^{\prime}, b^{\prime}\right) \times t \mapsto\left(x_{3}\left(a^{\prime} x_{0}^{2}-b^{\prime}\left(t x_{1}+x_{2}\right)^{2}\right), x_{3}\left(x_{1}-t x_{2}\right), x_{3}^{2}\right) .
\end{aligned}
$$

The coordinate transformations on the overlap st $=1, t a-s a^{\prime}=0$ and $b=b^{\prime}$ show that $F$ is isomorphic to $\mathrm{P}\left(\mathcal{O}_{p^{1}} \oplus \mathcal{O}_{\mathrm{p} 1}(1)\right)$. So $\tilde{D}$, too, is smooth of dimension 4 .

REMARK. This is another proof of the smoothness of $Z_{3}$.

Proposition 3.5. The map $\varphi: Z_{3} \rightarrow \mathscr{H}_{3}$ is an isomorphism.

Proof. Since $\varphi$ is birational and $\widetilde{\mathscr{H}}_{3}$ is irreducible, it is enough to show that the tangent map $d \varphi: \mathscr{T}_{Z_{3}}([C]) \rightarrow \mathscr{T}_{\tilde{\mathscr{H}}_{3}}([C / \Gamma])$ is injective in every point.

Consider a $\mathrm{C}^{*}$-action on $\mathrm{P}^{3}$ acting diagonally w.r.t. the coordinates $x_{0}, \ldots, x_{3}$ and having isolated fixed points. We can choose the action in such a way that the induced action on $\mathscr{H}_{3}$ has isolated fixed points, the fixed points being given by monomial ideals. Furthermore, the action leaves $Z_{3}$ invariant, hence descends to an action on $\tilde{\mathscr{H}}_{3}$ (more about torus actions in $\S 5$ ).

Suppose now that $[C] \in Z_{3}$ is a point where $d \varphi$ is not injective. Then $d \varphi$ is not injective in any point of $\mathrm{C}^{*} \cdot[C]$. Let $\left[C_{0}\right] \in \overline{\mathrm{C}^{*} \cdot[C]}$ be a fixed point in the closure. $d \varphi$ cannot be injective in $\left[C_{0}\right]$ neither, because then it would be injective on a whole neighborhood of $\left[C_{0}\right]$ which would contain also points of $\mathrm{C}^{*} \cdot[C]$.

Therefore it suffices to show that $d \varphi$ is injective in all points of $Z_{3}$ defined by monomial ideals. A sufficient condition for this to happen is given by the following.

LEMMA 3.6. Let $[C] \in Z_{3}$ be a point whose homogeneous ideal $I_{C}$ is generated by invariant monomials. Assume that there is no invariant monomial $m$ of degree 3 not contained in $I_{C}$ such that all monomials $x_{1} m, x_{2} m, x_{3} m$ lie in $I_{C}$. Then $d \varphi$ is injective in $[C]$.

We can check explicitly by looking at all types of monomial ideals, which we will determine in $\S 5$, that the assumption of the lemma is always met. This is a boring but simple exercise, and we will only point out the reasoning for one case.

Take for example the fixed curve $C$ with ideal $I_{C}=\left(x_{1} x_{2}, x_{1} x_{3}, x_{0}^{2} x_{2}\right)$, and let $m$ be a monomial of degree 3 not contained in $I_{C}$. Since $m$ is invariant, there are two possibilities:

(i) $x_{0}^{2}$ divides $m$. Then we have $m=x_{0}^{2} x_{i}, i=1$ or 3 , and $x_{i} m=x_{0}^{2} x_{i}^{2}$ is certainly not contained in $I_{C}$.

(ii) $x_{0}^{2}$ does not divide $m$. We have either $x_{1} \nmid m$, then $x_{2} m$ or $x_{3} m \notin I_{C}$, because not both can be multiples of $x_{0}^{2} x_{2}$; or $x_{1} \mid m$, then $m=x_{1}^{3}$, and $x_{1} m \notin I_{C}$. 
All other cases can be treated in a similar way. Thus $d \varphi$ is injective everywhere, and the proposition is proved.

It remains to prove the lemma.

Let $[C] \in Z_{3}$ be a point with homogeneous ideal $I_{C}=\left(h_{1}, \ldots, h_{r}\right)$, the $h_{i}$ being $\Gamma$-invariant monomials of degree $d_{i}$ (i.e., they contain $x_{0}$ in even power).

There is a natural injection

$$
\mathscr{T}_{\mathscr{H}_{3}}([C]) \subset \operatorname{Hom}\left(\mathscr{I}_{C}, \mathcal{O}_{C}\right) .
$$

The resolution of $\mathscr{I}_{C}$ defined by $I_{C}$ gives rise to a diagram

$$
\begin{aligned}
& \oplus_{i} \mathcal{O}_{\mathrm{P} 3}\left(-d_{i}\right) \stackrel{I_{\mathrm{C}}}{\longrightarrow} \mathscr{I}_{\mathrm{C}} \rightarrow 0 \\
& \vec{f} \mathscr{O}_{C}
\end{aligned}
$$

for every $f \in \operatorname{Hom}\left(\mathscr{I}_{C}, \mathcal{O}_{C}\right)$, and hence to an injective map

$$
\operatorname{Hom}\left(\mathscr{I}_{C}, \mathcal{O}_{C}\right) \stackrel{j}{\longrightarrow} \underset{i}{\oplus} \operatorname{Hom}\left(\mathcal{O}_{p^{3}}\left(-d_{i}\right), \mathcal{O}_{C}\right) \cong \underset{i}{\oplus} H^{o}\left(\mathcal{O}_{C}\left(d_{i}\right)\right)
$$

which sends $f$ to $\bar{f}$. Now let $I_{C}^{\prime}=\left(h_{1}^{\prime}, \ldots, h_{r^{\prime}}^{\prime}\right)$ be a homogeneous ideal of $C$ generated by monomials $h_{i}^{\prime}$ of even degree. In concrete terms, construct $I_{C}^{\prime}$ from $I_{C}$ by retaining the generators of even degree and replacing each generator $h_{i}$ of odd degree by generators $x_{0} h_{i}, \ldots, x_{3} h_{i}$. It is clear that $I_{C}$ and $I_{C}^{\prime}$ both define the same scheme $C$. Let

$$
\underset{i, \lambda}{\oplus} \mathcal{O}_{\mathrm{P}^{3}}\left(-d_{i, \lambda}^{\prime}\right) \stackrel{I_{C}^{\prime}}{\longrightarrow} \mathscr{I}_{C} \rightarrow 0
$$

be the corresponding surjection, where $d_{i, \lambda}^{\prime}=d_{i}+1$ and $\lambda=0, \ldots, 3$ if $d_{i}$ is odd and $d_{i, \lambda}^{\prime}=d_{i}$ and $\lambda=-1$ (say) if $d_{i}$ is even. By applying the exact functor $\left(\rho_{X_{*}}(\cdot)\right)^{\Gamma}$ to (3.7), we get a surjective map

$$
\bigoplus_{i, \lambda} \mathcal{O} \tilde{\mathrm{p}}\left(-d_{i, \lambda}^{\prime}\right) \stackrel{\left(\Phi\left(h_{i, \lambda}^{\prime}\right)\right)_{i, \lambda}}{\longrightarrow} \mathscr{I}_{C / \Gamma} \rightarrow 0 .
$$

But when $d_{i}$ is odd, then $\Phi\left(h_{i, 0}^{\prime}\right)=\Phi\left(x_{0} h_{i}\right)=0$, and we don't lose anything if we let the sum run only over indices $(i, \lambda)$ with $\lambda \neq 0$.

The diagram

$$
\begin{array}{cl}
\oplus_{i, \lambda} \mathcal{O}_{\mathrm{P}^{3}}\left(-d_{i, \lambda}^{\prime}\right) & \stackrel{I_{C}^{\prime}}{\longrightarrow} \mathscr{I}_{C} \rightarrow 0 \\
\downarrow^{\mu} & \| \\
\oplus_{i} \mathcal{O}_{\mathrm{P}^{3}}\left(-d_{i}\right) & \stackrel{I_{C}}{\longrightarrow} \mathscr{I}_{C} \rightarrow 0
\end{array}
$$

includes a diagram 


$$
\begin{array}{ccc}
\operatorname{Hom}\left(\mathscr{I}_{C}, \mathcal{O}_{c}\right) \stackrel{j}{\hookrightarrow} & \oplus_{i} H^{0}\left(\mathcal{O}_{c}\left(d_{i}\right)\right) \\
\| & \downarrow^{\kappa} \\
\operatorname{Hom}\left(\mathscr{I}_{C}, \mathcal{O}_{c}\right) \stackrel{j^{\prime}}{\hookrightarrow} & \oplus_{i, \lambda} H^{0}\left(\mathcal{O}_{c}\left(d_{i, \lambda}^{\prime}\right)\right) .
\end{array}
$$

The map $\kappa$ sends an element $(0, \ldots, \stackrel{i}{m}, \ldots, 0)$ to $(0, \ldots, \stackrel{(i,-1)}{m}, \ldots, 0)$ if $d_{i}$ is even and to $\left(0, \ldots, \stackrel{(i, 0)}{x_{0} m}, \ldots, \stackrel{(i, 3)}{\left.x_{3} m, \ldots, 0\right)}\right.$ otherwise.

The tangent space to $Z_{3}$ in $[C]$ is equal to $\left(\mathscr{T}_{\mathscr{H}_{3}}([C])\right)^{\Gamma}$, and there is a commutative diagram

$$
\begin{array}{ccc}
\mathscr{T}_{Z}([C]) & \hookrightarrow & \operatorname{Hom}\left(\mathscr{I}_{C}, \mathcal{O}_{C}\right)^{\Gamma} \\
\downarrow^{d \varphi} & & \downarrow^{\sigma} \\
\mathscr{T}_{\tilde{H}_{3}}([C / \Gamma]) & \hookrightarrow & \operatorname{Hom}\left(\left(\rho_{*} \mathscr{I}_{C}\right)^{\Gamma},\left(\rho_{*} \mathcal{O}_{C}\right)^{\Gamma}\right)
\end{array}
$$

(recall that $\mathscr{I}_{C / \Gamma}=\left(\rho_{*} \mathscr{I}_{C}\right)^{\Gamma}$ etc.). $\sigma$ is induced from the functor $\left(\rho_{X *}(\cdot)\right)^{\Gamma}$.

The last two diagrams fit together into a big dagram

$$
\begin{aligned}
& \mathscr{T}_{Z}([C]) \quad \hookrightarrow \quad \operatorname{Hom}\left(\mathscr{I}_{C}, \mathcal{O}_{C}\right)^{\Gamma} \stackrel{j}{\complement} \oplus_{i} H^{\mathrm{O}}\left(\mathcal{O}_{C}\left(d_{i}\right)\right)^{\Gamma} \\
& \downarrow \kappa \\
& \downarrow \sigma \oplus_{i, \lambda} H^{0}\left(\mathcal{O}_{C}\left(d_{i, \lambda}^{\prime}\right)\right)^{\Gamma} \\
& \downarrow \tau \\
& \mathscr{T}_{\tilde{\mathscr{H}}_{3}}([C / \Gamma]) \hookrightarrow \operatorname{Hom}\left(\left(\rho_{*} \mathscr{I}_{C}\right)^{\Gamma},\left(\rho_{*} \mathcal{O}_{C}\right)^{\Gamma}\right) \subset \underset{\substack{i, \lambda \\
\lambda \neq 0}}{i} H^{0}\left(\mathcal{O}_{C}^{\Gamma}\left(d_{i, \lambda}^{\prime}\right)\right) .
\end{aligned}
$$

The map $\tau$ simply forgets the components with indices $(i, 0)$ and is an isomorphism on the complement.

Now, in order to prove that $d \varphi$ is injective, it suffices to show that the composition $\tau \circ \kappa$ is injective.

By computing resolutions of all the ideals $\mathscr{I}_{C}$ (in Macaulay, for example), we see that they all are at least 3-regular, which means that $H^{p}\left(\mathscr{I}_{C}(m-p)\right)=0$ for all $p>0, m \geqq 3$; thus $H^{1}\left(\mathscr{I}_{c}(d)\right)=0$ for $d \geqq 2$. Therefore $H^{0}\left(\mathcal{O}_{c}\left(d_{i}\right)\right)^{\Gamma}$ is generated by the invariant monomials of $H^{0}\left(\mathcal{O}_{\mathrm{p}^{3}}\left(d_{i}\right)\right)$ not contained in $H^{0}\left(\mathscr{I}_{C}\left(d_{i}\right)\right)^{\Gamma}$.

Thus it is enough to check that no such monomial is contained in $\operatorname{ker}(\tau \circ \kappa)$. For monomials of even degree this is clear. because $\tau \circ \kappa$ is the identity on the direct summands $H^{0}\left(\mathcal{O}_{C}\left(d_{i}\right)\right)^{T}$ for $d_{i}$ even. An invariant monomial $m$ (which for short will stand for $(0, \ldots, m, \ldots, 0))$ of odd degree $d_{i}\left(d_{i}=3\right.$ in our case for all ideals) is mapped to $\left[\left(0, \ldots, x_{1} m, x_{2} m, x_{3} m, \ldots, 0\right)\right]$ by $\tau \circ \kappa$. Thus $\tau \circ \kappa(m)$ is zero exactly when $x_{1} m, x_{2} m, x_{3} m$ all lie in $H^{0}\left(\mathscr{I}_{C}\left(d_{i}+1\right)\right)^{T}$ (or, what amounts to the same, in $I_{C}$ ).

Now we are able to deduce the analogue to Theorem 3.3 (ii).

Proposition 3.7. $Z_{4}$ is isomorphic to $\tilde{\mathscr{H}}_{4}$, and $\tilde{\mathscr{H}}_{4}$ is a locally trivial fibration over $\check{\mathrm{P}}^{3}$ with fibers isomorphic to $\tilde{\mathscr{H}}_{3}$. Thus $\tilde{\mathscr{H}}_{4}$ is smooth of dimension 13. 
Proof. We can proceed as in the proof of Theorem 3.3 (ii).

A smooth rational quartic $C$ in $\mathrm{P}\left(2,1^{4}\right)$ spans exactly a hyperplane $H \cong \mathrm{P}\left(2,1^{3}\right)$ (i.e. a hypersurface with a linear equation $l\left(x_{1}, \ldots, x_{4}\right)$; use the fact that the lift of $C$ to $\mathrm{P}^{4}$ is an invariant elliptic quartic). Thus, the projection of $C$ to $\mathrm{P}^{3} \subseteq \mathrm{P}\left(2,1^{3}\right)$ from the singular point spans a hyperplane in $\mathrm{P}^{3}$, i.e. is a point in $\mathrm{P}^{3}$.

Again, no degeneration can possibly span less than a hyperplane because it then would already be contained in $\tilde{\mathscr{H}}_{3}$. But every degeneration in $\tilde{\mathscr{H}}_{3}$ spans the whole $\mathrm{P}\left(2,1^{3}\right)$. A semicontinuity argument shows again that no degeneration can span more than a hyperplane. Thus the map

$$
\begin{aligned}
\tilde{\mathscr{H}}_{4} & \rightarrow \check{\mathrm{P}}^{3} \\
{[C] } & \mapsto[\operatorname{span}(\operatorname{pr}(C))]
\end{aligned}
$$

is welldefined, and clearly a locally trivial fibration.

Consider now the map

$$
Z_{4} \rightarrow \tilde{\mathscr{H}}_{4} \rightarrow \check{P}^{3}
$$

which exhibits $Z_{4}$ as a locally trivial fibration over $\breve{p}^{3}$, with fiber $Z_{3}$. But $Z_{3}$ is isomorphic to $\tilde{\mathscr{H}}_{3}$, so $Z_{4}$ is isomorphic to $\tilde{\mathscr{H}}_{4}$.

\section{Rational quartics on Calabi-Yau hypersurfaces.}

From now on, we work over the ground field $C$.

A hypersurface in $\widetilde{P}=P\left(2,1^{4}\right)$ given by a polynomial of weighted degree 6 has trivial canonical bundle, i.e., is Calabi-Yau (the following is the only point where the Calabi-Yau comes into play).

Consider the ideal sequence of the universal family $p: \mathscr{C} \rightarrow \tilde{\mathscr{H}}_{4}$ of rational quartics in $\widetilde{P}$ :

$$
0 \rightarrow \mathscr{I}_{C} \rightarrow \mathcal{O}_{\tilde{\mathscr{H}}_{4} \times \tilde{\mathrm{P}}} \rightarrow \mathcal{O}_{\mathscr{C}} \rightarrow 0 .
$$

By twisting with $\mathcal{O}(6)$ and taking direct images under $p$, we get the sequence

$$
0 \rightarrow p_{*} \mathscr{I}_{C}(6) \rightarrow p_{*} \mathcal{O}_{\tilde{\mathscr{H}}_{4} \times \tilde{\mathrm{p}}}(6) \rightarrow p_{*} \mathcal{O}_{\mathscr{C}}(6) \rightarrow R^{1} p_{*} \mathscr{I}_{\mathrm{C}}(6) .
$$

If we assume for a moment that $R^{1} p_{*} \mathscr{I}_{C}(6)$ vanishes and that the (zeroth) direct images are locally free, this sequence reduces to

$$
0 \rightarrow p_{*} \mathscr{I}_{\mathscr{C}}(6) \rightarrow H^{0}\left(\mathcal{O}_{\tilde{\mathrm{p}}}(6)\right)_{\tilde{\mathscr{H}}_{4}} \stackrel{\rho}{\rightarrow} p_{*} \mathcal{O}_{\mathscr{C}}(6) \rightarrow 0 \text {. }
$$

Now take a section of $H^{0}\left(\mathcal{O}_{\tilde{p}}(6)\right)_{\tilde{\mathscr{H}}_{4}}$ which is induced from a generic section $F$ of

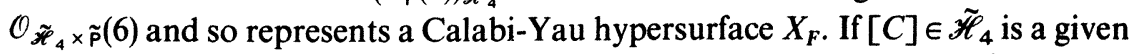
curve, then the induced section $\rho(F)$ of $p_{*} \mathcal{O}_{\mathscr{C}}(6)$ restricted to the fiber $H^{0}\left(C, \mathcal{O}_{C}(6)\right)$ over $[C] \in \tilde{\mathscr{H}}_{4}$ is equal to the restriction of $F$ to $C$. Hence, $\rho(F)$ vanishes exactly when $C$ is contained in $X_{F}$. 
Since the rank of $p_{*} \mathcal{O}_{\mathscr{C}}(6)$ equals the dimension of $\tilde{\mathscr{H}}_{4}$, Kleiman's Bertini theorem (cf. [9], Remark 6) implies that the zero scheme of the section $\rho(F)$ is finite and nonsingular; hence the length of this scheme is equal to the number of rational quartics on a generic Calabi-Yau hypersurface in $\widetilde{P}$. This number is given by the integral

$$
\int_{\tilde{\mathscr{H}}_{4}} c_{13}\left(p_{*} \mathcal{O}_{\mathscr{C}}(6)\right)
$$

$c_{13}$ being the top Chern class.

It remains to prove the claimed facts about the direct image sheaves.

PROPOSITION 4.1. $p_{*} \mathscr{I}_{\mathscr{C}}(6)$ and $p_{*} \mathcal{O}_{\mathscr{Q}}(6)$ are locally free sheaves, and $R^{1} p_{*} \mathscr{I}_{\mathscr{C}}(6)$ vanishes.

Proof. We show first that $H^{i}\left(\mathscr{I}_{C}(6)\right)=0$ for all curves $[C] \in \tilde{\mathscr{H}}_{4}, i \geqq 1$, and that $\operatorname{dim} H^{0}\left(\mathscr{I}_{C}(6)\right)$ is constant on $\widetilde{\mathscr{H}}_{4}$.

Since $\operatorname{dim} H^{i}\left(\tilde{\mathrm{P}}, \mathscr{I}_{C}(6)\right)$ is an upper semicontinuous function on $\tilde{\mathscr{H}}_{4}$, we have to show the vanishing of $H^{i}\left(\widetilde{P}, \mathscr{I}_{C}(6)\right)$ only for all degenerations with monomial ideals, and the constantness of $\operatorname{dim} H^{0}\left(\mathscr{I}_{C}(6)\right)$ for those and a generic curve.

Namely, let $[C] \in \tilde{\mathscr{H}}_{4}$ and suppose $\operatorname{dim} H^{i}\left(\mathscr{I}_{C}(6)\right)=d$. Take a one-dimensional torus action on $\tilde{P}$ such that the monomial curves are the fixed points of the action. For all $t \in \mathrm{C}^{*}$, the schemes $C_{t}:=t C$ are projectively equivalent, hence the cohomology groups have all the same dimension $d_{t}=d$. But the limit $C_{0}=\lim _{t \rightarrow 0} C_{t}$ is a monomial curve, and by semicontinuity, $\operatorname{dim} H^{i}\left(\mathscr{I}_{C_{0}}(6)\right) \geqq$ $\operatorname{dim} H^{i}\left(\mathscr{I}_{C}(6)\right)$.

As mentioned before, all the ideals of monomial degenerations of elliptic quartics $C^{\prime} \subseteq \mathrm{P}^{4}$ are at least 3-regular, thus $H^{i}\left(\mathrm{P}^{4}, \mathscr{I}_{C^{\prime}}(6)\right)=0$ for all $i>0$. By Lemma 2.1, it follows that $H^{i}\left(\widetilde{P}_{,} \mathscr{I}_{C}(6)\right)=0$ for all $i>0$ and all monomial degenerations of rational quartics. The constantness of $\operatorname{dim} H^{0}\left(\mathscr{I}_{C}(6)\right)$ can also be verified by an explicit computation.

Since $\mathcal{O}_{\mathscr{8}}(6)$ is flat over $\tilde{\mathscr{H}}_{4}, \mathscr{I}_{\mathscr{C}}(6)$ is a flat sheaf, too. By the previous result and cohomology and base change theorems ([7], III 12.11, 12.9) we conclude that $R^{1} p_{*} \mathscr{I}_{\mathscr{8}}(6)=0$ and that $p_{*} \mathscr{I}_{\mathscr{8}}(6)$ and $p_{*} \mathcal{O}_{\mathscr{8}}(6)$ are locally free.

\section{The calculation.}

We will calculate the integral (4.1) by Bott's formula, as follows (cf. [3] and [5]; these ideas are largely due to Geir Ellingsrud and Stein Arild Strømme):

Suppose we are given a $C^{*}$-action on $\tilde{P}_{\text {which }}$ induces a $C^{*}$-action with isolated fixed points on $\tilde{\mathscr{H}}_{4}$. This action in turn induces an equivariant $C *$-action on the tangent bundle $\mathscr{T}_{\tilde{x}_{4}}$ and on $p_{*} \mathcal{O}_{8}(6)$. Therefore, in a fixed point $x=[C]$ of the action, the respective fibers $\mathscr{T}_{\tilde{f}_{4}}(x)$ and $p_{*} \mathcal{O}_{\mathscr{8}}(6) \otimes \mathrm{C}(x)$ are $\mathrm{C}^{*}$-representations. 
As torus representations, they decompose into a direct sum of one-dimensional representations. Let $w_{1}(x), \ldots, w_{r}(x)$ resp. $\tau_{1}(x), \ldots, \tau_{r}(x)$ be the corresponding weights.

Then Bott's formula says in our context:

$$
\int_{\tilde{\mathscr{\#}}_{4}} c_{13}\left(p_{*} \mathcal{O}_{\mathscr{G}}(6)\right)=\sum_{x \in \tilde{\mathscr{H}}_{4}^{c *}} \frac{\tau_{1}(x) \cdot \ldots \cdot \tau_{r}(x)}{w_{1}(x) \cdot \ldots \cdot w_{r}(x)} .
$$

Let $T \subseteq \mathrm{GL}(5)$ be a maximal torus which acts diagonally on $\tilde{P}$ w.r.t. the coordinates $x_{0}, \ldots, x_{4}$ of $\mathrm{P}^{4}$. There are characters $\lambda_{0}, \ldots, \lambda_{4}$ on $T$ such that for any $t \in T$, we have $t \cdot x_{i}=\lambda_{i}(t) \cdot x_{i}$, and these characters generate the representation ring of $T$, i.e., if $W$ is a finite representation of $T$, we can write cum granum salis:

$$
W=\sum a_{p_{0} \ldots p_{4}} \lambda_{0}^{p_{0}} \cdot \ldots \cdot \lambda_{4}^{p_{4}} .
$$

The action of $T$ descends to an action on $\widetilde{P}=\mathrm{P}^{4} / \Gamma$.

In the following, we will compute the torus representations of the induced $T$-action on $\tilde{\mathscr{H}}_{4}$ in the fibers of $p_{*} \mathcal{O}_{\mathscr{C}}(6)$ and $\mathscr{T}_{\tilde{H}_{4}}$ in all fixed points. It is easy to see that a point $x \in \tilde{\mathscr{H}}_{4}$ is fixed exactly when the graded ideal of the corresponding curve is generated by monomials.

Then we choose a one-parameter subgroup $C^{*} \subseteq T$ with no non-trivial $\mathrm{C}^{*}$-weight in the tangent space of any fixed point. Such a one-parameter subgroup is given by a point $\left(w_{0}, \ldots, w_{4}\right)$ in the weight lattice $\operatorname{Hom}\left(C^{*}, T\right) \cong Z^{5}$; the corresponding characters on $C^{*}$ are given by $\lambda_{i}(t)=t^{w_{i}}$. If $\lambda_{0}^{p_{0}} \cdot \ldots \cdot \lambda_{4}^{p_{4}}$ is the character of the $C^{*}$-representation on an invariant one-dimensional subspace of the tangent space in a fixed point, the corresponding weight is given by

$$
w=p_{0} w_{0}+\ldots+p_{4} w_{4} .
$$

All these weights are nonzero if the weight vector $\left(w_{0}, \ldots, w_{4}\right)$ is chosen to avoid simultaneously all the (finitely many) hyperplanes in the weight lattice defined by the linear forms (5.2). Such a choice is clearly possible. In the concrete calculation of the integral (5.1) we try randomly chosen weights; if none of the denominators in the summands of (5.1) is zero - which would result in a "division by zero" error - the choice is valid.

Our choice of the weights guarantees that the $\mathrm{C}^{*}$-action on $\tilde{\mathscr{H}}_{4}$ has isolated fixed points (in fact, the same fixed points as the action of $T$ ), hence we will be able to apply Bott's formula.

We will first calculate the fixed points and tangent space representations for $\tilde{\mathscr{H}}_{3}$ and afterwards use the fact that $\tilde{\mathscr{H}}_{4}$ is a locally trivial fibration over $\tilde{P}^{3}$ with fiber $\tilde{\mathscr{H}}_{3}$. 
This is being done by calculating the fixed points of the $T$-action and the $T$-representation on the tangent spaces in each successive step of the blow up, i.e. on $\widetilde{G}$, on $\widetilde{G}^{\prime}$, and finally on $\tilde{\mathscr{H}}_{3}$ (Note that there are induced $T$-actions on those spaces and that fixed points lie over fixed points).

To compute the data of a blow up, say, of $X$ with center $B$, we have to get hold of the fiber $\mathscr{N}(x)$ of the normal bundle $\mathscr{N}=\mathscr{N}_{B / X}$ to the subvariety $B$ to blow up in each fixed point $x \in B$. We can achieve this by computing $T$-semiinvariant base vectors of $\mathcal{N}(x)$. Every such vector $\xi$ gives rise to a fixed point $x_{\xi}$ in the proper transform $B^{\prime}$ of $B: x_{\xi}$ is the inverse image of $x$ in the proper transform of a curve tangent to $\xi$ in $x$.

The tangent space at $x_{\xi}$ in the blow up $X^{\prime}=B l_{B} X$ is then given as

$$
\mathscr{T}_{X^{\prime}}\left(x_{\xi}\right) \cong L_{\xi} \oplus \mathscr{T}_{B}(x) \oplus \mathscr{T}_{\mathrm{P}\left(\mathcal{F}^{\vee}(x)\right)}\left(x_{\xi}\right),
$$

where $L_{\xi}$ is the span of $\xi$ in the normal space $\mathscr{N}(x)$. The isomorphism is equivariant. (Recall that the exceptional divisor of the blow up with center $B$ is isomorphic to the projective bundle $\mathrm{P}\left(\mathcal{N}_{B / X}^{\vee}\right)$, with normal space in $x_{\xi}$ isomorphic to $L_{\xi}$; cf. for instance [6], B. 6.)

Let us now look at the concrete calculations. We denote by $\widetilde{B^{\prime}}$ (resp. $\tilde{D^{\prime}}$ ) the proper transform of $\tilde{B}$ in $\tilde{G}$ (resp. of $\tilde{D}$ in $\tilde{\mathscr{H}}_{3}$ ).

Proposition 5.1. There are 126 fixed points in $\tilde{\mathscr{H}}_{3}$, and they are projectively equivalent to one of the following 25 types listed below. (To each fixed point type, we give the permutations of the variables which generate the remaining fixed points of that type.)

\begin{tabular}{|c|c|c|c|}
\hline lies in & fixed point type & permutations & $\begin{array}{l}\text { number of } \\
\text { fixed points }\end{array}$ \\
\hline$\tilde{G}-\tilde{B}$ & $\begin{array}{c}\left(x_{0}^{2}, x_{1}^{2}\right) \\
\left(x_{0}^{2}, x_{1} x_{2}\right) \\
\left(x_{1}^{2}, x_{2}^{2}\right) \\
\left(x_{1} x_{2}, x_{3}^{2}\right)\end{array}$ & $\begin{array}{c}\text { cyclic } \\
\text { permutations } \\
\text { of } \\
x_{1}, x_{2}, x_{3}\end{array}$ & $\begin{array}{l}3 \\
3 \\
3 \\
3\end{array}$ \\
\hline$\tilde{B^{\prime}}-\tilde{D}$ & $\begin{array}{c}\left(x_{1} x_{2}, x_{1} x_{3}, f\right) \\
f \in\left\{x_{0}^{2} x_{2}, x_{0}^{2} x_{3}, x_{2}^{3}, x_{2}^{2} x_{3}, x_{2} x_{3}^{2}, x_{3}^{3}\right\} \\
\left(x_{1}^{2}, x_{1} x_{2}, g\right) \\
g \in\left\{x_{2}^{3}, x_{2}^{2} x_{3}, x_{2} x_{3}^{2}, x_{0}^{2} x_{2}, x_{1} x_{3}^{2}, x_{1} x_{0}^{2}\right\}\end{array}$ & $\begin{array}{l}\quad x_{1} \leftrightarrow x_{2} \\
\text { and } x_{1} \leftrightarrow x_{3} \\
\text { all permutations } \\
\text { of } x_{1}, x_{2}, x_{3}\end{array}$ & $\begin{array}{l}6 \times 4 \\
3 \times 6\end{array}$ \\
\hline$\tilde{D^{\prime}}$ & $\begin{array}{c}\left(x_{1}^{2}, x_{1} x_{2}, x_{1} x_{3}^{2}, f\right) \\
f \in\left\{x_{2} x_{3}^{3}, x_{2}^{2} x_{3}^{2}, x_{3}^{4}, x_{2}^{4}, x_{2}^{3} x_{3}, x_{0}^{2} x_{2}^{2}\right\} \\
\left(x_{1}^{2}, x_{1} x_{2}, x_{1} x_{0}^{2}, g\right), \\
g \in\left\{x_{0}^{2} x_{2} x_{3}, x_{2}^{2} x_{3}^{2}, x_{0}^{4}, x_{2}^{4}, x_{2}^{3} x_{3}, x_{0}^{2} x_{2}^{2}\right\}\end{array}$ & $\begin{array}{c}\text { all } \\
\text { permutations } \\
\text { of } \\
x_{1}, x_{2}, x_{3}\end{array}$ & $\begin{array}{l}6 \times 6 \\
6 \times 6\end{array}$ \\
\hline
\end{tabular}


The tangent space in a fixpoint $x$ in $\tilde{G}$ is given by $\mathscr{T}_{\tilde{G}}(x)=\sum \lambda_{\gamma} \lambda_{\delta} \lambda_{\alpha}^{-1} \lambda_{\beta}^{-1}$, where $\alpha$, $\beta(\alpha \leqq \beta)$ run over the pairs of indices of the monomials in $I_{x}$, whereas $\gamma, \delta(\gamma \leqq \delta)$ run over all except these indices. The tangent space in each fixed point $x_{\xi}$ lying over a fixed point $x$ in $\tilde{B}$ resp. $\tilde{D}$ is calculated by formula (5.3), where the tangent spaces and normal spaces to $\tilde{B}$ resp. $\tilde{D}$ are given by:

\begin{tabular}{|c|c|}
\hline$I_{x_{\xi}}$ & tangent and normal spaces \\
\hline & \\
$\left(x_{1} x_{2}, x_{1} x_{3}, f\right)$ & $\mathscr{T}_{\tilde{B}}(x)=\lambda_{1} \lambda_{2}^{-1}+\lambda_{1} \lambda_{3}^{-1}+\lambda_{2} \lambda_{1}^{-1}+\lambda_{3} \lambda_{1}^{-1}$ \\
& $\mathscr{N}_{\tilde{B} / \tilde{G}}(x)=\lambda_{0}^{2} \lambda_{1}^{-1} \lambda_{2}^{-1}+\lambda_{0}^{2} \lambda_{1}^{-1} \lambda_{3}^{-1}+\lambda_{2} \lambda_{1}^{-1}+$ \\
& $\lambda_{3} \lambda_{1}^{-1}+\lambda_{3}^{2} \lambda_{1}^{-1} \lambda_{2}^{-1}+\lambda_{2}^{2} \lambda_{1}^{-1} \lambda_{3}^{-1}$ \\
& $\mathscr{T}_{\tilde{B}}(x)=\lambda_{3} \lambda_{1}^{-1}+\lambda_{3} \lambda_{2}^{-1}+\lambda_{2} \lambda_{1}^{-1}+\lambda_{3} \lambda_{1}^{-1}$ \\
& $\mathscr{N}_{\tilde{B} / \tilde{G}}(x)=\lambda_{0}^{2} \lambda_{1}^{-1} \lambda_{2}^{-1}+\lambda_{0}^{2} \lambda_{1}^{-2}+\lambda_{3}^{2} \lambda_{1}^{-1} \lambda_{2}^{-1}+$ \\
& $\lambda_{3}^{2} \lambda_{1}^{-2}+\lambda_{2} \lambda_{3} \lambda_{1}^{-2}+\lambda_{2}^{2} \lambda_{1}^{-2}$ \\
\hline & \\
& $\left.\mathscr{T}_{\tilde{D}}^{2}(x)=x_{1} x_{2}, g\right)$ \\
& $\mathscr{N}_{\tilde{D} / \tilde{G}}(x)=\lambda_{3} \lambda_{1}^{-1}+\lambda_{2} \lambda_{1}^{-1}+\lambda_{3} \lambda_{2}^{-1}+\lambda_{0}^{2} \lambda_{3}^{-2}$ \\
& $\lambda_{2}^{2} \lambda_{1}^{-1} \lambda_{3}^{-1}+\lambda_{2} \lambda_{1}^{-1}+\lambda_{0}^{2} \lambda_{2} \lambda_{1}^{-1} \lambda_{3}^{-2}$ \\
& \\
& $\mathscr{T}_{\tilde{D}}(x)=\lambda_{3} \lambda_{1}^{-1}+\lambda_{2} \lambda_{1}^{-1}+\lambda_{3} \lambda_{2}^{-1}+\lambda_{3}^{2} \lambda_{0}^{-2}$ \\
& $\mathscr{N}_{\tilde{D} / \tilde{G}}(x)=\lambda_{3} \lambda_{1}^{-1}+\lambda_{0}^{2} \lambda_{1}^{-1} \lambda_{2}^{-1}+\lambda_{2}^{3} \lambda_{1}^{-1} \lambda_{0}^{-2}+$ \\
& $\lambda_{2}^{2} \lambda_{3} \lambda_{0}^{-2} \lambda_{1}^{-1}+\lambda_{2} \lambda_{1}^{-1}+\lambda_{2} \lambda_{3}^{2} \lambda_{0}^{-2} \lambda_{1}^{-1}$ \\
\hline
\end{tabular}

Proof. The fixed points $x$ in the grassmannian $\widetilde{G}$ are readily determined, their ideals have the form $I_{x}=\left(x_{i} x_{j}, x_{k} x_{l}\right)$ with the obvious restrictions on the indices. Let $V_{2}:=H^{0}\left(\mathcal{O}_{\tilde{p}^{3}}(2)\right), V_{I_{x}}:=\mathrm{C} \cdot x_{i} x_{j} \oplus \mathrm{C} \cdot x_{k} x_{l}$. The tangent space in a fixed point $x$ is given by

$$
\mathscr{T}_{\tilde{G}}(x)=\operatorname{Hom}\left(V_{I_{x}}, V_{2} / V_{I_{x}}\right)=\sum \lambda_{\alpha}^{-1} \lambda_{\beta}^{-1} \lambda_{y} \lambda_{\delta},
$$

the indices being as specified in the proposition.

First blow up. The subvariety $\widetilde{B} \subseteq \tilde{G}$ to blow up consists of pencils with a fixed component, and the fixed points in $\tilde{B}$ are of type $\left(x_{1} x_{2}, x_{1} x_{3}\right)$ and $\left(x_{1}^{2}, x_{1} x_{2}\right)$.

Consider the fixed point $x$ with ideal $I_{x}=\left(x_{1} x_{2}, x_{1} x_{3}\right)$. The tangent space to $\tilde{G}$ in $x$ is given by

$$
\begin{aligned}
\mathscr{T}_{\tilde{G}}(x)= & \lambda_{1}^{-1} \lambda_{2}^{-1} \lambda_{0}^{2}+\lambda_{1}^{-1} \lambda_{3}^{-1} \lambda_{0}^{2}+\lambda_{2}^{-1} \lambda_{1}+\lambda_{3}^{-1} \lambda_{1}+2 \lambda_{1}^{-1} \lambda_{2}+ \\
& 2 \lambda_{1}^{-1} \lambda_{3}+\lambda_{1}^{-1} \lambda_{2}^{-1} \lambda_{3}^{2}+\lambda_{1}^{-1} \lambda_{3}^{-1} \lambda_{2}^{2} .
\end{aligned}
$$

First, we will determine a semiinvariant basis for the fiber $\mathscr{N}_{\tilde{B} / \tilde{\boldsymbol{G}}}(x)$ of the normal bundle of $\widetilde{B}$ in $\tilde{G}$. Let $\xi \in \mathscr{T}_{\tilde{G}}(x)$ be a semiinvariant tangent vector in $x$, given as $\xi=\left(\begin{array}{l}\xi_{1} \\ \xi_{2}\end{array}\right)$ w.r.t. the basis $\left\{x_{1} x_{2}, x_{1} x_{3}\right\}$ of $V_{I_{x}}$ (i.e., $\xi\left(a x_{1} x_{2}, b x_{1} x_{3}\right)=$ $\left.\left(a \xi_{1} x_{1} x_{2}, b \xi_{2} x_{1} x_{3}\right)\right)$. Since $\xi$ is semiinvariant, $\xi_{1}$ and $\xi_{2}$ are scalar multiples of 
a common Laurent monomial $\mu_{\xi}$ in $x_{0}, \ldots, x_{3}$ of degree 0 . Furthermore, the torus representation on the subspace spanned by $\xi$ is obtained by formally substituting $\lambda_{i}$ for $x_{i}, i=0, \ldots, 3$ in $\mu_{\xi}$. Clearly this monomial in the $\lambda_{i}$ has to be one of the summands in (5.4).

Now $I_{\xi}(t)=\left(x_{1} x_{2}+t \xi_{1} x_{1} x_{2}, x_{1} x_{3}+t \xi_{2} x_{1} x_{3}\right)$ is a curve through $x$ with tangent direction $\xi$ in $x$. We see that the semiinvariant tangent vectors

$$
\xi=\left(\begin{array}{c}
x_{1} x_{2}^{-1} \\
0
\end{array}\right),\left(\begin{array}{c}
0 \\
x_{1} x_{3}^{-1}
\end{array}\right),\left(\begin{array}{l}
x_{2} x_{1}^{-1} \\
x_{2} x_{1}^{-1}
\end{array}\right),\left(\begin{array}{l}
x_{3} x_{1}^{-1} \\
x_{3} x_{1}^{-1}
\end{array}\right)
$$

are tangent to $\widetilde{B}$; the curves given by $I_{\xi}(t)$ are even contained in $\tilde{B}$. The vectors

$$
\begin{gathered}
\left(\begin{array}{c}
x_{2} x_{1}^{-1} \\
-x_{2} x_{1}^{-1}
\end{array}\right),\left(\begin{array}{c}
x_{3} x_{1}^{-1} \\
-x_{3} x_{1}^{-1}
\end{array}\right),\left(\begin{array}{c}
x_{0}^{2} x_{1}^{-1} x_{2}^{-1} \\
0
\end{array}\right),\left(\begin{array}{c}
x_{3}^{2} x_{1}^{-1} x_{2}^{-1} \\
0
\end{array}\right), \\
\left(\begin{array}{c}
0 \\
x_{0}^{2} x_{1}^{-1} x_{3}^{-1}
\end{array}\right),\left(\begin{array}{c}
0 \\
x_{2}^{2} x_{1}^{-1} x_{3}^{-1}
\end{array}\right)
\end{gathered}
$$

complete the previous ones to a semiinvariant basis of $\mathscr{T}_{\tilde{G}}(x)$, thus they represent (modulo $\mathscr{T}_{\tilde{\mathbf{B}}}(x)$ ) a semiinvariant basis of $\mathscr{N}_{\tilde{\mathbf{B}} / \tilde{\boldsymbol{G}}}(x)$.

In order to compute the fixed points of $\tilde{G}^{\prime}$ lying over $x$, we consider the curves $I_{\xi}(t)$ for $\xi$ in that basis. Each of them defines a flat family of curves over $A^{1}-\{0\}$. We can extend this family in a unique way to a flat family over $A^{1}$. That flat family induces a map $A^{1} \rightarrow \tilde{\mathscr{H}}_{3}$ such that the image $x_{\xi}^{\prime}$ of $0 \in A^{1}$ maps onto a fixed point $x_{\xi}$ of $\tilde{G}^{\prime}$ under the blow up map $\tilde{\mathscr{H}}_{3} \rightarrow \tilde{G}^{\prime}$. The ideal $I_{x \xi}$ corresponding to $x_{\xi}$ is the subideal generated in degree 3 of the ideal corresponding to $x_{\xi}^{\prime}$ (cf. (3.2)).

To actually compute the ideal $I_{x \xi}$, we use a flattening algorithm described by Bayer and Mumford ([2], Ch. 1):

Proposition 5.2. Let $I(t)=\left(m_{1}(t), \ldots, m_{r}(t)\right)$ be the ideal of a family of schemes over $A^{1}$ and suppose that all $m_{i}:=m_{i}(0)$ are monomials. Let $I=\left(m_{1}, \ldots, m_{r}\right)$ be the ideal of the central fiber. Consider the following algorithm:

(i) Take a minimal syzygy of two generators $m_{i}, m_{j}$ of the ideal $I$, i.e., a relation

$$
h_{i} m_{i}+h_{j} m_{j}=0
$$

with $h_{i}$ and $h_{j}$ coprime monomials, which does not lift to a syzygy in $I_{\xi}(t)$. This means that we get a relation

$$
h_{i} m_{i}(t)+h_{j} m_{j}(t)=t^{s} g
$$

with $g \neq 0$ and $t \Varangle g$. Add the polynomial $g$ to the generators of $I(t)$ to get new ideals $I^{\prime}(t)$ and $I^{\prime}=I^{\prime}(0)$.

(ii) Repeat this process finitely often until all syzygies lift to syzygies in $I^{\prime}(t)$. 
Then the resulting ideal $I^{\prime}(t)$ defines a family of schemes which is flat in $t=0$, and the ideal of the central fiber is equal to $I^{\prime}$.

In our case, all $g$ 's are monomials and $s$ is equal to 1 in the first step. Furthermore, we are only interested in generators of degree 3 , so we can stop the iteration when we have added all monomials of degree 3 , and that is the case already after the first step, as can easily be seen.

In concrete terms, the only syzygy $x_{3} \cdot\left(x_{1} x_{2}\right)-x_{2} \cdot\left(x_{1} x_{3}\right)=0$ lifts to $I_{\xi}(t)$ as

$$
\begin{aligned}
x_{3} \cdot\left(x_{1} x_{2}+t \xi_{1} x_{1} x_{2}\right)-x_{2} \cdot\left(x_{1} x_{3}+t \xi_{2} x_{1} x_{3}\right) & =t \cdot\left(x_{3} \xi_{1} x_{1} x_{2}-x_{2} \xi_{2} x_{1} x_{3}\right) \\
& =: t \cdot f_{\xi} .
\end{aligned}
$$

All together, the fixed points in the blow up $\tilde{G}^{\prime}$ lying over $x$ are:

$$
I_{\xi}=\left(x_{1} x_{2}, x_{1} x_{3}, f_{\xi}\right), f_{\xi} \in\left\{x_{0}^{2} x_{2}, x_{0}^{2} x_{3}, x_{2}^{3}, x_{2}^{2} x_{3}, x_{2} x_{3}^{2}, x_{3}^{3}\right\},
$$

plus the ideals obtained by the permutations $x_{1} \leftrightarrow x_{2}$ and $x_{1} \leftrightarrow x_{3}$. The calculation for points $x$ of type $I_{x}=\left(x_{1}^{2}, x_{1} x_{2}\right)$ is exactly analogous.

Second blow up. In $\tilde{G}^{\prime}$, we have to blow up the non-flat locus $\tilde{D} \subseteq \tilde{G}^{\prime}$. The fixed points $x$ of $\tilde{G}^{\prime}$ contained in $\tilde{D}$ are of type $I_{x}=\left(x_{1}^{2}, x_{1} x_{2}, x_{1} x_{3}^{2}\right)$ and $I_{x}=$ $\left(x_{1}^{2}, x_{1} x_{2}, x_{1} x_{0}^{2}\right)$.

The tangent space to $\widetilde{G}^{\prime}$ in the first fixed point is given by (cf. (5.3)):

$$
\begin{aligned}
\mathscr{T}_{\tilde{G}}(x)= & 2 \lambda_{1}^{-1} \lambda_{3}+2 \lambda_{1}^{-1} \lambda_{2}+\lambda_{2}^{-1} \lambda_{3}+\lambda_{3}^{-2} \lambda_{0}^{2}+\lambda_{1}^{-1} \lambda_{2}^{-1} \lambda_{3}^{2}+ \\
& \lambda_{1}^{-1} \lambda_{3}^{-2} \lambda_{2}^{3}+\lambda_{1}^{-1} \lambda_{3}^{-1} \lambda_{2}^{2}+\lambda_{1}^{-1} \lambda_{3}^{-2} \lambda_{2} \lambda_{0}^{2} .
\end{aligned}
$$

We will determine (the torus representation of) the normal space $\mathcal{N}(x)$ to $\tilde{D}$ in $x$.

A curve in $\tilde{G}^{\prime}$ through $x$ tangent to $\xi \in \mathscr{T}_{\tilde{G}^{\prime}}(x)$ is given modulo $t^{2}$ as

$$
I_{\xi}(t)=\left(x_{1}^{2}+t \xi_{1} x_{1}^{2}, x_{1} x_{2}+t \xi_{2} x_{1} x_{2}, x_{1} x_{3}^{2}+t \xi_{3} x_{1} x_{3}^{2}\right),
$$

where $\xi=\left(\xi_{1}, \ldots, \xi_{3}\right)^{t}$, and if $\xi$ is semiinvariant, then the $\xi_{i}$ are scalar multiples of a common Laurent monomial $\mu_{\xi}$ of degree zero.

Again, by lifting a syzygy relation from $I_{x}$ to $I_{\xi}(t)$ (this can be done modulo $t^{2}$, too), we calculate the monomial $f_{\xi}$ we have to add to $I_{x}$ in order to get the fixed point in the blow up $\tilde{\mathscr{H}}_{3}$ corresponding to $\xi$. Since the syzygies of all pairs of monomials in $I_{x}$ generate the whole syzygy module, we only need to consider such pairs.

Suppose that lifting the syzygy of the pair $\left(m_{1}, m_{2}\right)$ results in the right monomial $f_{\xi}$; then $f_{\xi}$ is equal to $\mathrm{lcm}\left(m_{1}, m_{2}\right) \cdot \mu_{\xi}$. This monomial is supposed to have degree four. The two syzygies which can possibly yield a monomial of degree four are those between $x_{2}$ and $x_{1} x_{3}^{2}$ and between $x_{1} x_{2}$ and $x_{1} x_{3}^{2}$. Consider the first pair. We have $f_{\xi}=x_{1}^{2} x_{3}^{2} \cdot \mu_{\xi}$. But from (5.5) it is apparent that the Laurent monomials $\mu_{\xi}$ contain $x_{1}$ to the power -1 or higher, that means that $f_{\xi}$ contains $x_{1}$ as 
a factor. Hence the resulting ideal $I_{\xi}=\left(x_{1}^{2}, x_{1} x_{2}, x_{1} x_{3}^{2}, f_{\xi}\right)$ is contained in the ideal $\left(x_{1}\right)$ and certainly doesn't correspond to a flat degeneration.

So we only have to consider the pair $x_{1} x_{2}, x_{1} x_{3}^{2}$. We claim that the monomials $\mu_{\xi}$ corresponding to a semiinvariant basis of $\mathscr{N}(x)$ are

$$
\left\{x_{1}^{-1} x_{3}, x_{1}^{-1} x_{2}, x_{1}^{-1} x_{2}^{-1} x_{3}^{2}, x_{1}^{-1} x_{3}^{-2} x_{2}^{3}, x_{1}^{-1} x_{3}^{-1} x_{2}^{2}, x_{1}^{-1} x_{3}^{-2} x_{2} x_{0}^{2}\right\}
$$

The two other possibilities for $\mu_{\xi}$, namely $x_{2}^{-1} x_{3}$ and $x_{3}^{-2} x_{0}^{2}$, are excluded because they again lead to ideals contained in the ideal $\left(x_{1}\right)$. On the other hand, $\mathscr{N}(x)$ has dimension 6; together this proves the claim.

So, the fixed points in the blow up $\tilde{\mathscr{H}}_{3}$ lying over $I_{x}=\left(x_{1}^{2}, x_{1} x_{2}, x_{1} x_{3}^{2}\right)$ are

$$
I_{\xi}=\left(x_{1}^{2}, x_{1} x_{2}, x_{1} x_{3}^{2}, f_{\xi}\right), \quad f_{\xi} \in\left\{x_{2} x_{3}^{3}, x_{2}^{2} x_{3}^{2}, x_{3}^{4}, x_{2}^{4}, x_{2}^{3} x_{3}, x_{0}^{2} x_{2}^{2}\right\} .
$$

The torus representation of $\mathcal{N}(x)$ is gotten by formally summing up the monomials in (5.6) and substituting $\lambda$ for $x$. To get all fixed points, we have to add the fixed points generated by all permutations of the variables $x_{1}, x_{2}$ and $x_{3}$. The calculation for points $x$ of type $I_{x}=\left(x_{1}^{2}, x_{1} x_{2}, x_{1} x_{0}^{2}\right)$ is again analogous.

Now we have determined all fixed points of the torus action on $\tilde{\mathscr{H}}_{3}$ : they are the fixed points of all stages of the blow up process minus the fixed points lying in the blow up loci.

Fixed points and tangent spaces in $\tilde{\mathscr{H}}_{4}$. A fixed curve in $\tilde{\mathrm{P}}$ spans a $T$-invariant hyperplane $\left(\cong P\left(2,1^{3}\right)\right)$ in $\tilde{P}$, which is given by an equation $x_{i}=0, i \in\{1, \ldots, 4\}$. Thus, if for instance $i=4$, we get the ideals of all fixed curves lying in $\left\{x_{4}=0\right\}$ by adjoining the monomial $x_{4}$ to the ideals previously determined. The ideals of the remaining fixed points are obtained by cyclically permuting the variables $x_{1}, \ldots, x_{4}$ in these ideals. Thus, $\widetilde{\mathscr{H}}_{4}$ containes 504 fixed points all together.

According to Proposition $3.7, \tilde{\mathscr{H}}_{4}$ is fibered over $\check{\mathrm{P}}^{3}$ as

$$
\begin{aligned}
& \pi: \tilde{\mathscr{H}}_{4} \rightarrow \check{P}^{3} \\
& {[C] \quad \mapsto\left[\text { projection of } \operatorname{span}(C) \text { to } \mathrm{P}^{3} \subseteq \tilde{\mathrm{P}}\right] . }
\end{aligned}
$$

The tangent space of $\tilde{\mathscr{H}}_{4}$ in a fixed point $x=[C]$ therefore decomposes equivariantly as a direct sum

$$
\mathscr{T}_{\tilde{\mathscr{\#}}_{3}}(x) \oplus \mathscr{T}_{\check{\mathrm{p}}_{3}}(\pi(x)) .
$$

Let $C$ be an invariant curve spanning the singular hyperplane $\left\{x_{i}=0\right\}$ and let $V_{i}=\mathrm{C} \cdot x_{i}, V=\mathrm{C} \cdot x_{1} \oplus \ldots \oplus \mathrm{C} \cdot x_{4}$. Then the torus representation of the tangent space to $\mathscr{P}^{3}$ is equal to

$$
\mathscr{T}_{\check{\rho}_{3}}(\pi(x))=\operatorname{Hom}\left(V_{i}, V / V_{i}\right)=\sum_{j \neq i} \lambda_{j} \lambda_{i}^{-1}
$$

Finally, the torus representations on the fibers $H^{0}\left(\mathcal{O}_{c}(6)\right)$ of $p_{*} \mathcal{O}_{\mathscr{C}}(6)$ are easily 
determined: $H^{0}\left(\mathcal{O}_{C}(6)\right)$ is spanned by those monomials of degree 6 that don't lie in the ideal $\mathscr{I}_{C}$.

The MAPLE-program which implements these computations is available from the author upon request.

\section{REFERENCES}

1. Dan Avritzer and Israel Vainsencher, Compactifying the space of elliptic quartic curves, London Math. Soc. Lecture Notes Ser. 179, 46-58.

2. Dave Bayer and David Mumford, What can be computed in algebraic geometry? Preprint.

3. Raoul Bott, A residue formula for holomorphic vector fields, J. Differential Geom. 1 (1967), 311-330.

4. Igor Dolgachev, Weighted projective varieties, Group Actions and Vector Fields, Lecture Notes in Math. 956, 34-77.

5. Geir Ellingsrud and Stein Arild Strømme, The number of twisted cubic curves on Calabi-Yau complete intersections, Lectures given at Dyrkolbotn August 10-14, 1993.

6. William Fulton, Intersection Theory, Springer, 1984.

7. Robin Hartshorne, Algebraic Geometry, Springer, 1977.

8. Sheldon Katz, Rational curves on Calabi-Yau manifolds: verifying predictions of mirror symmetry, Preprint OSU-M-92-3.

9. Steven L. Kleiman, The transversality of a general translate, Compositio Math. 28 (1974), 287-297.

10. David Morrison, Picard-Fuchs equations and mirror maps for hypersurfaces, Essays on Mirror Manifolds, International Press, pp. 241-264.

11. David Mumford, Lectures on Curves on an Algebraic Surface, Amer. Math. Soc. 59.

DEPARTMENT OF MATHEMATICS

UNIVERSITY OF BERGEN

N-5007 BERGEN

NORWAY

E-mail address: Paul.Meurer@mi.uib.no 